\title{
Athalie (1690) de Racine à la lumière des sources hébraïques et grecques : La lutte des sacralités
}

\section{Adrien Paschoud}

\section{(2) OpenEdition}

\section{Journals}

Édition électronique

URL : http://journals.openedition.org/edl/397

DOI : $10.4000 /$ edl.397

ISSN : 2296-5084

Éditeur

Université de Lausanne

\section{Édition imprimée}

Date de publication : 15 mai 2010

Pagination : 189-204

ISBN : 978-2-940331-22-2

ISSN : 0014-2026

Référence électronique

Adrien Paschoud, "Athalie (1690) de Racine à la lumière des sources hébraïques et grecques : La lutte des sacralités », Études de lettres [En ligne], 1-2 | 2010, mis en ligne le 15 mai 2013, consulté le 18

décembre 2020. URL : http://journals.openedition.org/edl/397 ; DOI : https://doi.org/10.4000/edl.397 


\section{ATHALIE (1690) DE RACINE À LA LUMIÈRE DES SOURCES HÉBRAÏQUES ET GRECQUES : LA LUTTE DES SACRALITÉS}

Euvre de commande destinée aux chastes pensionnaires de Saint-Cyr, Athalie (1690) marque la fin de la carrière théâtrale de Racine. Inspirée des grands textes de l'Ancien Testament, notamment le Livre des Rois, cette pièce puise également dans un vaste ensemble de sources grecques dont Ion d'Euripide. Tragédie du "schisme" (Roland Barthes), Athalie réforme dans le sens de la foi un matériau antique disparate offert aux variations sur la mort, le pouvoir, la filiation, l'origine obscure et l'élection. Plus précisément, la tragédie fait grand usage du mysterium tremendum que suggère le Dieu de l'Ancien Testament: "terreur", "horreur», "tremblement», mais aussi "ravissement» et "éblouissement» sont autant de termes qui se rapportent à une fascination pour ce que nous nommerions aujourd'hui le sacré. En peignant non sans ambiguïtés la lutte qui oppose Dieu et les dieux, Athalie présente un rapport différentiel avec le tragique.

Athalie, Tragédie tirée de l'écriture sainte (1690) marque, on le sait, le terme de la carrière théâtrale de Racine. Les circonstances qui ont présidé à l'élaboration de cette tragédie biblique sont bien connues. Commandée par le Roi, l'œuvre a été conçue pour l'édification des demoiselles de Saint-Cyr sous la tutelle bienveillante de Mme de Maintenon. Elle succède de peu à Esther dont le succès fut retentissant. Racine s'engage alors dans un projet qu'il juge plus ambitieux et dont il résume ainsi l'intrigue: "[Athalie] a pour sujet Joas reconnu et mis sur le trône" ${ }^{1}$. Plus étendue qu'Esther, Athalie doit répondre à un double impératif:

I. Racine, «Préface» d'Athalie, in Euvres complètes, p. 872. Toutes les références à Athalie seront citées d'après cette édition. 
d'une part, respecter les canons aristotéliciens tels que les doctes les avaient définis, et susciter ainsi la crainte et la pitié (catharsis); d'autre part, provoquer la ferveur religieuse en faisant montre de la puissance de Dieu. Paroles et chants élaborent de fait une poétique «sublime» aux seules fins de "ravir", au sens esthétique et religieux, le chaste public de Saint-Cyr ${ }^{2}$. Le matériau antique - qu'il soit hébraïque ou grec - sert bien évidemment de pivot à cette entreprise d'édification.

Les sources sur lesquelles repose Athalie ont été amplement documentées. Celles-ci sont multiples et s'enchevêtrent souvent. Bien qu'une obscure Athalia ait été représentée en latin dans le collège jésuite de Clairmont en 1658, la tragédie de Racine est fondée d'abord et avant tout sur une lecture attentive de l'Ancien Testament et plus particulièrement du Livre des Rois (II 11 et 12) et du Livre des Chroniques (II 24) ${ }^{3}$. L'Ancien Testament relate comment Joas, petit-fils d'Athalie, fut sauvé in extremis des mains de son aïeule (Athalie entendait venger la mort d'Okosias en tuant sa propre descendance). Caché dans le temple de Jérusalem, Joas fut ensuite mis sur le trône tandis qu'Athalie, attirée par le prêtre Joad dans un guet-apens, était mise à mort. Les textes vétérotestamentaires ne s'attardent guère sur le règne sanglant d'Athalie; sa mort n'est qu'une simple étape dans l'apothéose que constitue le couronnement de Joas. Celui-ci marque en effet le rétablissement de l'alliance rompue entre Dieu et son peuple. Outre l'Ancien Testament, Racine a lu l'historien Flavius Josèphe qu'il cite dans la préface d'Athalie et dont il s'inspire pour le dénouement de la pièce. Dans ses Antiquités judä̈ques, l'historien romain comble en partie les lacunes laissées par le texte biblique (celui-ci reste en définitive peu loquace à propos de la mort d'Athalie) et se montre ainsi soucieux de reconstituer l'enchaînement cohérent des événements. Racine fera de même. La part prédominante de l'Ancien Testament dans la genèse d'Athalie n'exclut pas d'autres sources, issues de l'Antiquité grecque. Celles-ci ne sont cependant guère nombreuses: sans doute faut-il mentionner le théâtre tragique d'Eschyle, notamment Les Perses, et celui d'Euripide, notamment Ion

2. Au XVII e siècle, le mot "ravir» désigne en effet l'éblouissement esthétique; il désigne également l'élévation mystique, l'union avec Dieu et l'enlèvement violent. Voir C. Reichler, "Scènes du ravissement".

3. On n'oubliera pas ce que Racine doit également à l'Histoire universelle de Bossuet dans laquelle l'épisode biblique d'Athalie est évoqué. 
(dont on peut penser que Racine a suivi en partie le canevas et auquel il a emprunté certaines scènes, notamment le dialogue de Créuse et d'un jeune serviteur d'Apollon, qui est en réalité son fils).

Par le fait qu'il soit conjointement précis et lacunaire, le texte biblique se prête remarquablement aux grands principes de la poétique et de la tragédie sacrées. Tout le travail de Racine consistera en une amplification de ses sources: il devra concilier fidélité (le poète dramatique ne peut prendre que des libertés très restreintes par rapport au texte source) et invention (il s'agit de combler les manques du texte source, mais surtout d'adapter celui-ci aux attentes du public et à son code éthique, sans toutefois heurter les principes édictés par les doctes). On peut distinguer au moins trois procédés mis en œuvre par Racine, qui respectent tous la Poétique d'Aristote. En premier lieu, Racine amplifie la menace qui pèse sur le jeune Joas en dépit (ou à cause) du fait qu'il soit caché; Athalie se livre à une traque inquisitoriale pour découvrir l'identité du jeune enfant. En second lieu, Racine exploite le procédé traditionnel, très en vogue au XVII ${ }^{e}$ siècle, du songe prémonitoire ${ }^{4}$ (Athalie voit dans son rêve un enfant qui la menace et la tue); le topos aristotélicien de l'enfant caché et de l'origine obscure est ensuite exploité sous l'angle de l'absence et de la présence, du visage et du masque, de l'ignorance et du savoir (ainsi Athalie ignore-t-elle que Joas est en réalité son petit-fils avant la scène de reconnaissance, qui scelle sa mort imminente). Enfin, en troisième lieu, Racine crée un dénouement au cours duquel le héros caché triomphe devant l'usurpatrice. Le dernier acte d'Athalie respecte le renversement des actions, considéré par Aristote comme étant le meilleur type d'action tragique: celui qui veut faire mourir meurt, tandis que son adversaire est sauvé. C'est du reste un procédé conventionnel que Racine avait déjà mis en ouvre dans Mithridate et Iphigénie.

Par rapport à Esther, qui suivait scrupuleusement le récit biblique, Athalie prend quelques libertés sur le plan de la composition. Alors qu'Esther était construite sur deux journées et était constituée de trois tableaux, Athalie respecte plus rigoureusement la règle des trois unités propre, on le sait, à la dramaturgie classique. Reposant sur une action unique, l'intrigue se déroule en quelques heures dans un même lieu. Plus encore, Racine utilise habilement les ressources de la tragédie alternée dont Esther avait constitué une première ébauche. Dans Athalie, les

4. Voir G. Forestier, "Le rêve littéraire du baroque au classicisme». 
chants du chœur se substituent aux entractes et assurent une rigoureuse continuité scénique: Racine précise en effet dans sa "Préface» qu' [il a] aussi essayé d'imiter des anciens cette continuité d'action qui fait que leur théâtre ne demeure jamais vide» et que «les intervalles des actes ne [sont] marqués que par des hymnes et par des moralités du chœur, qui ont rapport à ce qui se passe " 5 . Il y a donc coïncidence temporelle entre ce qui se produit dans le hors-scène et les louanges et les craintes que le chœur adresse à Dieu. Racine fait coïncider sans heurts le temps de l'action et le temps de la représentation. Athalie accède à cette «illusion mimétique", à cet idéal esthétique que prônent les doctes et dont l'objectif est de faire en sorte que le spectateur oublie qu'il est au théâtre. Tout cela est au service de la seule histoire véritable, celle qui est voulue et dictée par Dieu. Racine exploite ainsi les ressorts traditionnels de la tragédie sainte: dialogues, évocations du passé, tableaux animés (hypotypose), contenu du rêve, prophéties, chants du cœur, etc. ; autant d'éléments qui concourent bien évidemment à la glorification de Dieu. La tragédie sera du reste louée par les contemporains de Racine, notamment Boileau, lequel y voit la marque du sublime:

Tout ce qui peut y avoir de sublime paraît rassemblé dans ces quatre vers [v. 61-64]: la grandeur de la pensée, la noblesse du sentiment, la magnificence des paroles, et l'harmonie de l'expression si heureusement terminée par ce dernier vers [...]. D’où je conclus que c'est avec très peu de fondement que les admirateurs outrés de M. Corneille veulent insinuer que M. Racine lui est beaucoup inférieur pour le sublime $^{6}$.

La critique moderne s'est quant à elle beaucoup interrogée sur le sens à donner, du point de vue théologique, à Athalie. Les liens étroits, mais également conflictuels, qui ont uni Racine et Port-Royal ont conduit certains commentateurs à voir dans Athalie une pièce janséniste (il faut rappeler que Racine, à partir de 1677, renonce au théâtre et se rapproche de ses anciens maîtres). L'Antiquité hébraïque d'Athalie ne serait en quelque sorte que la transposition de l'anthropologie théologique de

5. Racine, «Préface» d'Athalie dans Euvres complètes, p. 874.

6. Boileau, Réflexions, dans Euvres complètes, p. 562. Dans la "Préface» d'Esther, Racine relève la "manière sublime dont [les grandes vérités de l'Ecriture] sont énoncées" (Euvres complètes, p. 812). 
Port-Royal, fondée sur la corruption irrémissible de l'homme et sur la prédestination ${ }^{7}$. Seul le salut par la grâce importe dans un univers qui a vu la séparation d'un Dieu omnipotent et d'une créature viciée par le péché originel. Pourtant, à y regarder de près, cette lecture se heurte à plusieurs écueils: elle supposerait tout d'abord que le jansénisme soit un courant religieux homogène, caractérisé par une ligne doctrinale forte, et dont la fiction théâtrale traduirait de manière mimétique l'essence. Or, on sait aujourd'hui que sous cette dénomination se cache une multitude d'individus et de divergences théologiques. De plus, les liens entre Racine et Port-Royal demeurent lâches et il est sans doute inexact, comme le pense Philippe Sellier, de voir dans Athalie l'œuvre d'un homme qui chercherait à se convertir ${ }^{8}$. Si l'on peut admettre que le jansénisme de Racine est "sentimental, familial et social», et non pas "doctrinal» ${ }^{9}$, il faut se méfier d'une "théorie du reflet» qui superposerait imprudemment l'homme et l'œuvre. Par ailleurs, sur un plan plus strictement théologique, une lecture attentive d'Athalie suggère que le Dieu qui y est représenté n'est pas le Dieu de la prédestination ni de l'humanité pécheresse. En fait, Athalie reprend, sans le modifier, le paradoxe biblique selon lequel Dieu conduit tout à sa fin en laissant aux hommes le soin de se conduire eux-mêmes. Dans cette optique, Dieu ne s'est nullement retiré du monde comme le montrent en permanence les miracles qu'il accomplit; ce sont les hommes qui s'égarent et qui se détournent de lui au point de s'entretuer ou d'adorer de faux dieux; ce sont eux qui se livrent aux pires turpitudes intellectuelles et physiques, tels les libertins, les pyrrhoniens et autres sectateurs d'Epicure, cette "troupe impie" (v. 820) qui vit dans une dangereuse insouciance et que la tragédie, via le chœur, stigmatise. Seule la foi véritable guide l'homme.

On perçoit dès lors quel peut être le gain de cette interprétation du paradoxe divin dans une perspective tragique, qui allie, on le sait, l'humain (ethos) et le divin (daimon). Tout est explicable en termes purement humains et politiques: Athalie, à l'image d'Agrippine, cherche à asseoir définitivement sa domination en écrasant le dernier foyer de résistance; pure incarnation de la libido dominandi, elle entend instaurer la religion rivale, celle de Baal, dont le prêtre apostat Mathan est

7. C'est la thèse développée par J. Orcibal dans La Genèse d' "Esther» et d' "Athalie».

8. Ph. Sellier, «Le jansénisme des tragédies de Racine».

9. R. Picard, De Racine au Parthénon, p. 47. 
l'infâme représentant. Ce faisant, elle permet au futur roi de révéler son identité et de rétablir la lignée brisée de David. Mais tout est également exprimable en termes religieux: c'est Athalie qui, persuadée d'avoir été guidée par une puissance supérieure, s'écrie d'elle-même: «Impitoyable Dieu, toi seul as tout conduit» (v. 1774). Cette lecture est cautionnée par les paroles inspirées du Grand Prêtre, Joad, et par les interventions du chœur qui chante Dieu et la manière dont il mène l'Histoire. Racine va au-delà des apories de la condition humaine pour donner sens à l'histoire, pour l'expliquer et la justifier. Les personnages d'Athalie, bons et mauvais, sont inscrits dans une sorte de psychomachie allégorique; ils sont destinés à faire entrevoir la puissance et la justice immanente de Dieu. Cette perspective providentialiste est légitimée de manière également paradoxale par la seconde prophétie présente dans Athalie qui veut que Joas, après un règne heureux, abandonne Dieu et fasse assassiner Zacharie dans le temple. Avant même de mourir Athalie lance cette terrible imprécation:

\section{[...] je me flatte, j'espère}

Qu'indocile à ton joug, fatigué de ta loi,

Fidèle au sang d'Achab, qu'il a reçu de moi,

Conforme à son aïeul, à son père semblable,

On verra de David l'héritier détestable

Abolir tes honneurs, profaner ton autel,

Et venger Athalie, Achab et Jézabel ${ }^{10}$.

Racine ne fait que suivre fidèlement le texte biblique et s'en explique ainsi :

[L]e meurtre [de Zacharie] commis dans le temple fut une des principales causes de la colère de Dieu contre les Juifs, et de tous les malheurs qui leur arrivèrent dans la suite. On prétend même que depuis ce jour-là les réponses de Dieu cessèrent entièrement dans le sanctuaire. C'est ce qui m’a donné lieu de faire prédire tout de suite à Joad et la destruction du temple et la ruine de Jérusalem. Mais comme les prophètes joignent d'ordinaire les consolations aux menaces, et que d'ailleurs il s'agit de mettre sur le trône un des ancêtres du Messie, j’ai

Io. Racine, Athalie, v. 1784-1790. 
pris occasion de faire entrevoir la venue de ce consolateur, après lequel tous les anciens justes soupiraient ${ }^{11}$.

La chute de Joas, prédite par Athalie, ne pouvait surprendre le public de la fin du XVII ${ }^{\mathrm{e}}$ siècle. Celui-ci sait que le Livre des Rois et le Livre des Chroniques retracent la longue succession des rois pieux et des rois apostats, puis la ruine de Jérusalem et la captivité de Babylone. Il ne faut donc pas par conséquent subordonner l'intrigue d'Athalie à une forme d'ironie tragique, celle-là même qui repose sur le modèle archétypique d'Edipe de Sophocle (EEdipe ignore que le coupable qu'il cherche et qu'il entend implacablement punir n'est autre que lui-même). Tout au plus pourrait-on dire que cette configuration est évidée de sa substance pour être réformée dans le sens de la foi. De fait, il est également inexact de voir une disqualification du modèle providentialiste. Rappelons que certains écrivains et commentateurs modernes ont évoqué une "épiphanie négative" et ont posé l'hypothèse selon laquelle Athalie constitue un gigantesque leurre, qui se plairait à miner de manière souterraine le message chrétien qu'elle exalte. Ainsi Jean Giraudoux écrivait-il que: "[Racine] a enfin trouvé une fatalité, plus impitoyable que la fatalité antique ${ }^{12}$. Aussi séduisante soit-elle, cette interprétation est sans doute anachronique; elle prête à Racine des intentions particulièrement subversives qui le rapprocheraient des stratégies textuelles mises en œuvre par certains représentants du "libertinage érudit» au XVII siècle ${ }^{13}$.

Le meurtre perpétré par Joas doit davantage se lire à la lumière des deux temporalités qui traversent Athalie: l'une religieuse et linéaire (les hommes vivent dans l'attente de l'accomplissement de la promesse divine); l'autre historique et cyclique (l'action des hommes n'a de sens qu'en référence à un modèle ancien que les monarques reproduisent). En peignant l'inévitable dégradation que constitue l'histoire des hommes lorsque ceux-ci s'éloignent de Dieu, Athalie repose sur le sentiment d'une perte qui donne rétroactivement à l'homme le sens de son existence; elle évoque en filigrane le christianisme de l'Eglise primitive, celui que les

II. Racine, «Préface» à Athalie, p. 875.

I2. J. Giraudoux, «Introduction», dans Racine, Athalie, p. 41. Michel Butor propose une lecture semblable: Athalie et, dans une moindre mesure, Esther constitueraient une violente remise en question du christianisme (voir "Racine et les dieux»).

13. Voir C. Venesoen, "Athalie ou le demi-échec de la théologie tragique», p. 30-31. 
hommes du XVII e siècle finissant ont appelé de leurs vœux devant la faillite des valeurs et la corruption de la Cour ${ }^{14}$. La tragédie de Racine réfracte peut-être les excès de l'absolutisme ${ }^{15}$, ou du moins invite à une réflexion sur les processus de rationalisation politique qui préside à la figure royale, celle-ci n'opérant plus sur le plan de la sacralité mais sur le plan du politique, comme le suggère Jean-Marie Apostolidès dans son ouvrage Le Prince sacrifié. Théâtre et politique au temps de Louis XIV:

Cet acte [de rationalisation politique] créera une situation de séparation: au grand-prêtre reviendra le domaine du sacré et du sacrifice, au roi celui du gouvernement des hommes. Celui-ci aura son origine dans la trahison des serments, la mauvaise foi et le refus du sacrifice ${ }^{16}$.

L'impossible retour à une origine perdue est figurée par un réseau particulièrement dense d'oppositions entre le sacré et le profane, entre le pur et l'impur, entre l'unicité et la pluralité, entre l'immanence et la transcendance. Elle met en scène deux figures sacrificielles: l'une négative, Athalie, l'autre positive, Joas. Une opposition qui se manifeste par la collusion de deux univers de représentation, l'un antique, l'autre biblique, le premier se subordonnant au second afin de faire surgir la puissance de Dieu par la figuration d'images propres à frapper l'esprit du public (c'est le procédé de l'hypotypose). Ainsi le vers 1667 ("Grand Dieu, voici ton heure, on t'amène ta proie») fait-il écho aux vers 1038-1040: "Les chiens, à qui son bras a livré Jézabel / Attendant que sur toi sa fureur se déploie, / Déjà sont à la porte et demandent leur proie». La mort d'Athalie est en quelque sorte une propriation offerte au Dieu de l'Ancien Testament afin de marquer l'accomplissement de la Promesse.

La tragédie de Racine demeure inféodée, on le voit, à une charpente providentialiste, sur laquelle vient se greffer une réflexion de nature historique et politique. Les intentions apologétiques reposent sur une opposition somme toute convenue entre le judéo-christianisme et le paganisme antique. On saura aisément s'en convaincre en relevant les nombreuses occurrences des termes "profanation", "sacrilège», "impiété », «idoles»,

I4. Voir B. Neveu, Erudition et religion aux XVII et XVIII e siècles, p. 333-363 ( L'érudition ecclésiastique du XVII siècle et la nostalgie de l'Antiquité chrétienne»).

15. Certaine commentateurs y ont vu une allusion masquée à la Révocation de l'Edit de Nantes (1685).

I6. J.-M. Apostolidès, Le Prince sacrifié, p. 131. 
"faux dieux", etc. Largement représenté dans la pièce, le paganisme antique acquiert pourtant une dimension qui le situe bien au-delà de sa fonction première, celle de servir de repoussoir aux vérités bibliques. Il apparaît dès lors comme un remarquable embrayeur. En effet, et bien qu'il soit condamné et réduit à un vain simulacre, le paganisme antique induit un questionnement, une fascination trouble, une zone d'interférences, par le fait qu'il constitue un objet de scandale, au sens étymologique du terme. Athalie et le prêtre apostat Mathan incarnent cette sacralité pervertie mais qui les dote d'un véritable pouvoir d'inversion ou de transgression des signes. Dès la première scène de la pièce, Mathan est présenté comme un personnage fourbe, un impie, un parjure, celui qui "veut anéantir le Dieu qu'il a quitté» (v. 42). Outre le fait qu'il incarne une figure machiavélique, Mathan est un démiurge, un être qui crée des mondes: "Pour vous perdre, il n'est point de ressorts qu'il n'invente" (v. 43). C'est un homme qui incarne le détournement des discours qu'il se plaît à se réapproprier: "Le Ciel est juste et sage et ne fait rien en vain ", dit-il, parodiant le discours chrétien (v. 558). Mathan est celui qui invalide tout rapport transparent entre les mots et les choses. Il infléchit les discours, les vide de leur substance et use remarquablement de la ductilité des mots (v. 933-936):

J'approchai par degrés de l'oreille des rois,

Et bientôt en oracle on érigea ma voix.

J'étudiai leur cœur, je flattai leurs caprices,

Je leur semai de fleurs le bord des précipices.

N'est-ce pas là le casuite qui s'exprime dont la morale a paru si dévoyée aux yeux des jansénistes? Ennemi juré du "Dieu des juifs", Mathan interroge le rôle dévolu au langage dans l'énonciation de la croyance et des procédures de fabrication et d'accréditation de celle-ci.

Le dialogue entre Athalie et Joas (acte II, scène VII) met également en scène le dévoiement des actions et surtout des discours. Désirant attirer à elle Joas, Athalie use de tous les artifices. Elle se révèle être une figure redoutable, faisant miroiter au jeune enfant une bien séduisante existence: "Venez dans mon palais, vous y verrez ma gloire. / (...) Les plaisirs près de moi vous chercheront en foule» (v. 679 et 687). Aux yeux du public du XVII ${ }^{\mathrm{e}}$ siècle, ces paroles ne pouvaient évoquer autre chose que les passages néo-testamentaires dans lesquels le Diable tente à plusieurs reprises le Christ. Mais un pas supplémentaire est franchi 
lorsqu'Athalie propose une possible conciliation des sacralités: «J'ai mon Dieu que je sers. Vous servirez le vôtre. / Ce sont deux puissants Dieux" (v. 683-684). Il y a là un scandale qui ne laisse de stupéfier le jeune Joas: la théologie et la morale se heurtent au monde, à sa corruption, à sa morale "toute païenne». Surtout, il y a en filigrane un questionnement relatif à la norme et à son application. Aux yeux d'Athalie, la morale chrétienne ne recouvre pas le champ de tous les possibles: aussi est-ce la respecter que d'aménager les voies de son application et d'en nuancer les modalités. Athalie fait du monothéisme chrétien un système parmi d'autres; soucieuse peut-être de gouverner sagement, elle se montre accueillante aux contraires, absorbe des croyances dont la coexistence paraît impossible et s'offre en intercesseur entre deux univers de croyance. Sur un autre plan, on aura deviné derrière l'ascétisme de Joas une manière détournée d'attaquer les jésuites qui ont eux-mêmes attaqué le rigorisme janséniste: il n'est pas nécessaire pour être un saint, ou un dévot, d'être "sans yeux pour les beautés de l'art et de la nature", ni, lors des jours de fête de se retirer "parmi les morts " ${ }^{17}$. Moins d'un quart de siècle après la publication des Provinciales de Pascal et des intenses polémiques qu'elles ont suscitées, les jansénistes ne pouvaient que se sentir flattés par cette condamnation implicite de leurs plus farouches adversaires.

Cette confusion des signes, sciemment induite par Athalie ou Mathan, trouve son origine dans ce que j'appellerais une déchirure ontologique. Dans des pages suggestives, Roland Barthes soutient qu'Athalie est une tragédie sacrée qui s'intéresse avant tout à ceux qui se tiennent du côté des dieux païens: "Il existe chez Racine, on le sait, une contradiction entre son éthique et son esthétique: le Bien, qu'il choisit, est chez lui une abstraction, mêlée de conformisme, ses personnages appremment positifs sont des personnages ennuyeux, des sortes de grands masques vides; le Mal qu'il condamne est vivant; sous la noirceur apparente, des nuances, des tentations, des regrets s'agitent, comme si dans le héros noir

I7. "L'honneur et la gloire sont des idoles qu'il ne connaît pas, et pour lesquelles il n'a point d'encens à offrir. Une belle personne lui est un spectre. Et ces visages impérieux et souverains, ces agréables tyrans qui font partout des esclaves volontaires et sans chaînes, ont le même pouvoir sur ses yeux que le soleil sur ceux des hiboux", Le Moyne, Peintures morales, cité dans la lettre IX des Provinciales de Pascal (p. 146). Voir également le Tartuffe de Molière (III, 3, 933 et suivants). 
venait se déposer le noyau même de la subjectivité racinienne ${ }^{18}$. Figure quasiment prométhéenne, Mathan a un passé, il a effectué un parcours religieux qui l'individualise:

Moi seul, donnant l'exemple aux timides Hébreux,

Déserteur de leur loi, j’approuvai l'entreprise,

Et par là de Baal méritai la prêtrise.

Par là je me rendis terrible à mon rival,

Je ceignis la tiare, et marchai son égal ${ }^{19}$.

Les motivations avancées par Mathan (il s'éloigne de ce qu'il révère en raison du seul attrait des richesses; son orgueil démesuré l'incite à vouloir égaler Dieu) ne peuvent toutefois masquer une conscience déchirée:

Toutefois, je l'avoue, en ce comble de gloire,

Du Dieu que j’ai quitté l'importune mémoire

Jette encore en mon âme un reste de terreur;

Et c'est ce qui redouble et nourrit ma fureur ${ }^{20}$.

La présence de ce mysterium tremendum révèle l'être dans ses hésitations et dans ses contradictions: seule l'apostasie rend possible la singularité. De même que Mathan, Athalie est un personnage dont la subjectivité est exacerbée. En proie à des visions oniriques d'une grande violence, caractérisée par "l'inquiétude» (v. 896), elle n'est plus maîtresse d'ellemême; son impuissance à mener à bien son projet politique reflète son incapacité à comprendre le monde. L'exemple le plus célèbre et le plus souvent commenté est le songe dans lequel Athalie voit apparaître sa mère Jézabel:

Je l'évite partout, partout il me poursuit.

C'était pendant l'horreur d'une profonde nuit.

Ma mère Jézabel devant moi s'est montrée,

Comme au jour de sa mort pompeusement parée.

Ses malheurs n'avaient point abattu sa fierté.

Même elle avait encore cet éclat emprunté,

Dont elle eut soin de peindre et d'orner son visage,

Pour réparer des ans l'irréparable outrage.

I8. R. Barthes, Sur Racine, p. 129.

19. Racine, Athalie, v. 950-954.

20. Racine, Athalie, v. 955-959. 
Tremble, m'a-t-elle dit, fille digne de moi.

Le cruel Dieu des Juifs l'emporte aussi sur toi.

Je te plains de tomber dans ses mains redoutables, Ma fille. En achevant ces mots épouvantables,

Son ombre vers mon lit a paru se baisser.

Et moi, je lui tendais les mains pour l'embrasser.

Mais je n'ai plus trouvé qu'un horrible mélange

D'os et de chair meurtris, et traînés dans la fange,

Des lambeaux pleins de sang, et des membres affreux,

Que des chiens dévorants se disputaient entre eux ${ }^{21}$.

Fondé sur des sources à la fois antiques et modernes (Les Perses d'Eschyle, La Jérusalem délivrée du Tasse, La Mariane de Tristan L'Hermite, Horace et Polyeucte de Corneille, etc.), ce récit, dont Claude Simon se souviendra dans La Route des Flandres, offre une collusion entre la beauté et l'abjection, la tendresse et la souffrance, la nomination et l'indicible, le pur et l'impur; il s'insère dans la tradition du baroque macabre du premier XVII" siècle. C'est un "tableau " qui est offert ici au spectateur et dont la fonction est de s'imprimer dans l'esprit de ce dernier. On aura bien entendu reconnu l'attribut rhétorique de l'hypotypose: l'évocation de l'objet est plus forte que l'objet lui-même. Athalie fait de la lutte des sacralités le support d'une poétique nocturne, fondé sur le principe de réversion des images. On voit aisément quel parti Racine a pu tirer de la force agissante des images et des effets qu'elles génèrent dans l'esprit troublé d'individus "furieux" ou «inquiets». Le tragique racinien se pense bien entendu comme substance édifiante, mais il n'exclut aucunement un engagement de l'âme, une méditation sur la puissance des passions. Surtout, le tragique racinien se meut dans les interstices du profane et du sacré, et joue sur des frontières sémantiques nécessairement floues. La lutte des sacralités est donc le prétexte à une exploration des possibles liés à l'imagination, cette "puissance trompeuse» dénoncée par les moralistes du XVII e siècle. Plus encore, la figure menaçante de Joas s'apparente en quelque sorte à une fatalité intérieure ou intériorisée, non pas transcendante mais immanente. La très riche extension tragique que Racine a donnée à sa conception du Dieu chrétien n'invalide pas la trame apologétique qui sous-tend Athalie, mais elle constitue une sorte

2I. Racine, Athalie, v. 490-507. 
de point aveugle, une sphère dans laquelle se meuvent les soubresauts de l'ethos.

$\mathrm{Au}$ terme de ce parcours, plusieurs constats doivent retenir notre attention. Athalie marque la coprésence à la fois pacifique et conflictuelle de l'antiquité hébraïque et grecque, la première se subordonnant à la seconde. Construite sur trois thèmes (la vengeance, la purification par le biais du sacrifice et la manifestation de Dieu par l'entremise des miracles), Athalie se prête remarquablement aux schèmes de la tragédie antique tant du point de vue de son ancrage théorique (via les interprétations d'Aristote) que de sa composante politique et surtout anthropologique (la tragédie est une cérémonie, un lieu où se rencontre l'humain et le divin ${ }^{22}$ ). Tous ces éléments se voient évidemment réformés dans le sens de la foi. Pourtant, Athalie laisse par endroits apparaître la menace sourde que constitue un autre système de croyances, le culte des «faux dieux", qui est présenté comme une alternative certes dévoyée, mais éminemment puissante. Le paganisme antique apparaît en effet comme une tentative avortée de substituer à la Vérité un ensemble de vérités. C'est au fond la productivité des "fables" qui est mise en lumière et dont la charge subversive, à peine énoncée, est immédiatement désamorcée ${ }^{23}$. D'une certaine manière la lutte des sacralités relève davantage d'une lutte des discours relatifs à la croyance; elle réfracte métaphoriquement la figure du poète dramatique. Ce grand architecte fait d'une trame apologétique, somme toute banale, le lieu où des forces s'affrontent au sein d'une matière poétique qui allie l'ordre et le désordre, l'unicité et la pluralité, la stabilité des signes et leur inquiétante prolifération sémantique.

Adrien PAschoud

Fonds national suisse de la recherche scientifique

22. Voir J. Scherer, Racine etlou la cérémonie.

23. A l'acte III, scène III, l'égarement de Mathan touche au burlesque. 


\section{BIBLIOGRAPHIE}

\section{Textes}

Pascal, Les Provinciales, édité par Michel Le Guern, Paris, Folio, 1987. Racine, Athalie, in Euvres complètes, édité par Raymond Picard, tome 1, Paris, La Pléiade, 1950.

-, Athalie, Paris, Grasset, 1931.

Boileau, Euvres complètes, édité par Antoine Adam, Paris, La Pléiade, 1966.

\section{Etudes}

Apostolidès, Jean-Marie, Le Prince sacrifié. Théâtre et politique au temps de Louis XIV, Paris, Minuit, 1985.

Barthes, Roland, Sur Racine, Paris, Seuil, 1963 (1960).

Butor, Michel, "Racine et les dieux", in Répertoire I, Paris, Minuit, 1961, p. 28-60.

Forestier, Georges, "Le rêve littéraire du baroque au classicisme: réflexes typologiques et enjeux esthétiques", Revue des Sciences humaines, 211/3 (1988), p. 213-235.

Neveu, Bruno, Erudition et religion aux XVII et XVIII e siècles, Paris, Albin Michel, 1994.

Orcibal, Jean, La Genèse d' "Esther" et d' "Athalie", Paris, Vrin, 1950.

PiCard, Raymond, De Racine au Parthénon. Essai sur la littérature et l'art à l'âge classique, Paris, Gallimard, 1977.

Reichler, Claude, "Scènes du ravissement", in La Littérature et le brillant, éd. A. Chamayou, Arras, Artois Presses Université, 2002, p. 29-41.

Scherer, Jacques, Racine etlou la cérémonie, Paris, PUF, 1982. 
Sellier, Philippe, «Le jansénisme des tragédies de Racine: réalité ou illusion?», CAEIF, 31 (1979), p. 135-148.

Venesoen, Constant, "Athalie ou le demi-échec de la théologie tragique", in Racine. Mythes et réalités, Société d'étude du XVII ${ }^{\mathrm{e}}$ siècle, London (Canada), Université de Western Ontario, 1976, p. 25-51. 
